\title{
SISTEMA DE PRODUCCIÓN A PEQUEÑA ESCALA DE DULCE DE LECHE CAPRINO EN COSTA RICA ${ }^{1}$
}

\author{
David Mora Valverde ${ }^{2}$
}

\begin{abstract}
RESUMEN
Sistema de producción a pequeña escala de dulce de leche caprino en Costa Rica. El objetivo de este trabajo fue implementar un estudio de factibilidad para la diversificación productiva y comercial de un sistema caprino a través de la producción de dulce de leche. Éste incluyó el estudio de factibilidad, el desarrollo del proceso de producción y el diseño de producto para la elaboración a pequeña escala. Fue desarrollado en la provincia de San José, Costa Rica, en el cantón de Mora entre los meses de agosto del 2009 hasta enero del 2010. Se cuantificaron datos por concepto de inversión, costos de producción, e índices financieros. El estudio obtuvo una inversión de US \$1360 ( $\varnothing 685000 *$ ) para una unidad con capacidad de transformación de 50 litros diarios. Se definió con un costo de producción por litro de dulce de leche de US\$ 6,46 ( $\$ 3265)$, por presentación de $275 \mathrm{ml}$ un costo de US\$2,54 ( $₫ 1284)$. Con una tasa de actualización de $12 \%$ la proyección del proyecto generó un valor actual neto (VAN) de US\$ 24530 ( $\$ 12388$ 000) (US\$ 0,41 ó ф209 por unidad de venta), con un periodo de vida útil del mismo estimado en diez años. Conjuntamente se determinó un valor de Tasa interna de retorno (TIR) de $226 \%$. Respecto a los costos productivos anuales, el rubro de mayor importancia representó la compra de leche, con un 35\% del costo total de producción, seguido por el envasado (18\%) y etiquetado (16\%). El dato de rentabilidad financiera correspondió a un 17,9\%.
\end{abstract}

\begin{abstract}
Small-scale production system of goat milk caramel in Costa Rica. The aimed of this study was conduct a technical and practical factibility study to assess and describe the possibilities of diversification of a goat milk project through the milk caramel production. This included the feasibility study, production process and product design on a small scale system in the province of San José, Costa Rica, in the canton of Mora between the months of August 2009 to January 2010. Data were obtained by concept of investment, production costs, and financial ratios. The project obtained an investment of US $\$ 1360$ ( $\varnothing 685000^{*}$ ) for a processing unit with capacity of 50 liters per day. A production cost per liter of fresh milk of US\$ 6,46 ( $\$ 3265$ ) was determined, for a presentation of $275 \mathrm{ml}$ and price of US\$2,54 ( $\not 1284)$. Setting an actualization rate of $12 \%$, the projection generated an actual net value (NPV) of US\$ $24530(\$ 12,388,000)$ (US\$ 0,41 or $₫ 209$ per unit sold), with an estimated life span of ten years. An Internal Rate of Return (IRR) of $226 \%$ was also determined. Regarding the annual production costs, the most important item was purchase of milk, with $35 \%$ of the total cost of production, followed by packaging $(18 \%)$ and labeling $(16 \%)$. The profitability data corresponded to $17.9 \%$.
\end{abstract}

Key words: Goats, milk processing, profitability, agri business

Palabras clave: Procesamiento de leche, caprinocultura, cabras, rentabilidad, agronegocios.

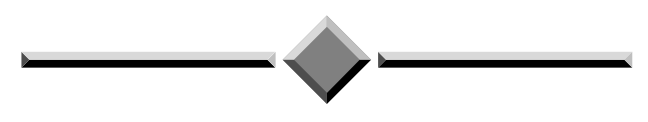

\footnotetext{
1 Recibido: 7 de octubre, 2011. Aceptado: 12 de marzo, 2012. La investigación pertenece al proyecto 737-A8-155. Inscrito ante la Vicerrectoría de Investigación. Universidad de Costa Rica.

2 Estación Experimental Alfredo Volio Mata. Facultad de Ciencias Agroalimentarias. Universidad de Costa Rica. david.mora@ucr.ac.cr

* 505 colones equivalen a un dólar norteamericano (tipo de cambio Noviembre del 2010).
} 


\section{INTRODUCCIÓN}

La producción de leche de cabra (Capra hircus) en Costa Rica, representa una oportunidad de emprendimiento atractiva cuando exista firmeza y evidencia previa en la incursión de mercados claramente definidos. Por encontrarse la actividad en una etapa incipiente y sensible, demanda mecanismos facilitadores que conyeven a una incursión más sólida respecto a fugaces iniciativas en el pasado, donde se estimule la correcta incursión comercial a través del uso de mejores técnicas de producción primaria y la generación valor agregado en la cadena productiva que generen oportunidades económicas, para comunidades locales (Mora 2010).

En la actualidad, una amplia variedad de productos lácteos son ofrecidos en los centros de venta masivos. Los avances tecnológicos de los últimos años han permitido desarrollar nuevos productos lácteos muchos de los cuales tienen demostrados efectos sobre la salud humana (Castillo y Taberna 2000). Por ello, entre otros, el carácter globalizado del comercio ha permitido efectuar explotaciones capaces de suplir la demanda de estos mercados exigentes y distantes tal y como ya se da en nuestro país con diversas marcas de derivados lácteos y cárnicos. Para el dulce de leche de cabra, conocido a nivel latinoamericano como arequipe (Colombia), manjar (Perú), cajeta (México), el cual en algunos casos si no es elaborado a partir de leche de cabra, no pueden ostentar el título correspondiente de su país (SE 2005) en Costa Rica ha sido un producto desarrollado a pequeña escala sin comercialización formal ni mayor conocimiento en la población (Chacón 2010) donde lo más cercano se muestra en la disponibilidad en el mercado nacional de una golosina denominada "cajeta a base de leche caprina", el cual es importado desde México y no se encuentra disponible en todo momento (Mendez 2010).

La diversificación en la industrialización de productos caprinos permite cierta constancia en el flujo de capital para el productor lechero de tal manera que en épocas de alta disponibilidad lechera (bajos precios) exista la opción de aumentar a través del procesamiento, la durabilidad de los bienes obtenidos, lo cual permitiría para efectos de comercialización, contar con mayor flexibilidad de tiempo. Lo anterior sugiere que el proceso de agregar valor a un producto primario puede ir como opción clara para los productores de tal manera que esto aumente su independencia comercial. Esto permite visualizar proyectos con riesgos externos menores siempre y cuando se incluya la evaluación financiera de la adición de valor agregado a la materia prima a través de distintos productos lácteos donde se muestren los potenciales beneficios económicos para el productor caprino (Mora 2010).

Se espera ofrecer un marco de referencia para identificar los costos de producción del dulce de leche de cabra en Costa Rica, en una estructura de carácter pequeño que apunta como eje central hacia la articulación del apoyo comercial y financiero como modelo empresarial para la capacitación y el mejoramiento de la transferencia de conocimientos en la transformación de derivados lácteos de cabra.

El objetivo de este trabajo fue implementar un estudio de factibilidad en la diversificación productiva de su sistema zootécnico hacia la producción de dulce de leche caprino maximizando sus características de valor agregado.

\section{MATERIALES Y MÉTODOS}

Este proyecto formó parte de un acompañamiento técnico efectuado a un productor caprino por los responsables del proyecto de investigación "Análisis Bioeconómico para el establecimiento de explotaciones caprinas" ligado a la Estación Experimental Alfredo Volio Mata de la Universidad de Costa Rica en Ochomogo, Cartago. La investigación se implementó en la provincia de San José, en el Cantón de Mora. Fue efectuado entre los meses de agosto del 2009 hasta enero del 2010.

El acompañamiento técnico al productor caprino nació a partir de la idea y necesidad de ofrecer un producto procesado que le permitiese a éste diversificarse logrando flexibilidad ante eventuales fluctuaciones en los precios de compra de la leche caprina por parte de procesadores, cuando existe sobre oferta así como para encontrar una opción de conservación de la leche cuando existen remanentes no comercializados. El trabajo incluyó el desarrollo de ensayos para determinación de costos de producción, la asesoría en la adquisición de equipo y utilización de materias primas para el proceso, acondicionamiento de las áreas de trabajo para efectuar el producto con estándares mínimos 
y/o reglamentarios de calidad (Encalada et al. 1999, INTI 2008, MEIC 2002) así como el asesoramiento en el diseño de producto y su presentación.

El producto específico fue clasificado como Dulce de Leche sin Agregados (Zunino 2000), el cual contiene elementos básicos para su elaboración (leche entera $100 \%$ de cabra, azúcar de caña y bicarbonato de sodio), sin la adición de fuentes espesantes. Desde el inicio se tomó esta decisión con la finalidad de elaborar un producto de considerable pureza a partir de leche de alta calidad (acidez no mayor a 18 grados Dornic, control de temperaturas máximas (Chacón 2004)), donde se remarcara el procesamiento artesanal como valor agregado, el cual fue uno de los ejes directos para el acompañamiento en la aplicación de bases conceptuales del diseño de un producto alimenticio turístico (Barbosa 2007) con tres ejes de caracterización del valor agregado del producto que le dieran identidad comercial propia, los cuales son: 1. Materia prima (leche) proveniente de un hato manejado acorde con estándares internacionales en bienestar animal (HFAC 2005). 2. Elaborado con una receta artesanal y 3. Proveniente de una micro empresa local resaltando valores de sostenibilidad ambiental y social.

A partir de la ejecución de siete pruebas piloto utilizando en promedio 30 litros de leche de cabra, se definieron las características generales del producto terminado como tal: presentación de $275 \mathrm{ml} 80$ grados Brix, rendimiento promedio de $35 \%$ (leche entera/dulce de leche) y la proporción de ingredientes mostrada en el Cuadro 1.

El diseño del producto para una presentación de $275 \mathrm{ml}$ en envase de vidrio, implicó la adaptación de requisitos generales de etiquetado (MEIC 2002, SE 2006) la presentación del mismo así como el proceso de diseño de imagen de marca para el producto.

Cuadro 1. Proporción de ingredientes utilizada para la elaboración del dulce de leche de cabra. San José, Costa Rica. 2010.

\begin{tabular}{lc}
\hline Materias primas & Porcentaje de inclusión \\
\hline Leche & $82 \%$ \\
Azúcar & $18 \%$ \\
Bicarbonato de Sodio* & $0,08 \%$ \\
Sorbato de Potasio* & $0,01 \%$ \\
TOTAL & $\mathbf{1 0 0 \%}$ \\
\hline
\end{tabular}

*Grado alimentario.
Se determinaron costos de inversión para los siguientes rubros: adquisición de equipo para la elaboración y control de proceso: Acidímetro, recipientes de acero inoxidable, filtros de malla fina, filtros para producto terminado, utensilios varios (removedores, cucharas), refractómetro, quemador de gas y adaptación de una zona para el procesamiento. La inversión en refrigeración no se toma en cuenta debido a que la unidad caprina ya contaba con este equipo. De igual manera se determinaron las siguientes variables para la ejecución del flujo de caja en el cual la unidad se percibió como una división independiente donde el subproceso de elaboración de dulce de leche adquiere la materia prima al productor (a sí mismo). Se efectuó de esta manera para darle más independencia al objetivo del ensayo así como eliminar posibles subestimaciones en los precios de la leche, por lo tanto en los costos de operación, lo que fijó un precio actualizado de US\$1,08 ( $\varnothing 550$ colones) (Mora 2010) por litro. Las otras variables calculadas fueron: mano de obra, costos por empaque, etiquetado, consumo de energía (gas LP), costos de distribución, cálculos de precios a distribuidores y precios sugeridos a terceros expendedores, debido a que, aunque el volumen de leche procesado es pequeño, la cantidad de unidades a mercado de $275 \mathrm{ml}$ y sus características de valor agregado (mayor precio) puede exceder la capacidad de comercialización basándose en el área de influencia comercial del productor. Los precios de mercado fueron actualizados hasta octubre del 2010 y el volumen productivo estimado correspondió al procesamiento de dos lotes semanales de 50 litros, lo cual corresponde a un promedio del $30 \%$ de la producción de la unidad caprina sujeto de este estudio y un total de 400 litros mensuales de leche.

Los cálculos económicos se efectuaron a través de la metodología INTA (Sabadzija et al. 2007), donde se definieron, cuantificaron y valoraron los bienes de capital requeridos para el modelo propuesto. Posteriormente, proyectando a un periodo de diez años, se estimaron los resultados económicos potenciales del procesamiento mediante el margen bruto (Ingresos Brutos - Costo directo); el costo de producción total y por litro de dulce de leche. El resultado económico del establecimiento como conjunto de proyecto se calculó mediante el valor de Ingreso neto (IN) anual y la rentabilidad financiera. La evaluación financiera de la inversión se estimó (a 10 años, sin crecimiento de la producción anualmente) mediante el valor actual neto 
(VAN) y la tasa interna de retorno (TIR) utilizando el método de tanteos (Benavides 2007) usando una tasa de actualización del $12 \%$ donde se definió anualmente un incremento en los costos de producción de un $10 \%$ igualando en la misma escala el precio de venta del producto.

\section{RESULTADOS Y DISCUSIÓN}

Conociendo que el análisis teórico para evaluar las posibilidades de un proyecto permite que el mismo pueda estar apoyado en indicadores técnicos (Nastasi 2007) y concibiendo un proyecto como un agroecosistema, donde éste es la unidad de análisis, y su contexto, donde se observan la totalidad de factores que lo integran para poder llegar a una descripción de la autonomía del mismo (Vilaboa et al. 2006) y aunado a un plan estratégico, como motor de todo el proceso de planificación y también operativo, indica la vía a seguir para formalizar las distintas estrategias y luego verificar y rendir cuenta de su factibilidad (Nastasi 2007).

En el proceso de creatividad productiva, uno de los retos más interesantes para las personas y empresas vinculadas a una actividad y sus sub-actividades, es la creación de nuevos productos de los cuales se espera que sean novedosos, pertinentes y rentables en el amplio espacio de la oferta y la demanda (Barbosa 2007).

El dulce de leche para la presente investigación es entendido como el producto obtenido por concentración y acción del calor a presión normal o reducida de la leche, con o sin adición de sólidos de origen láctico y/o crema y adicionado de sacarosa (parcialmente sustituido o no por monosacáridos y/u otros disacáridos) con o sin adición de otras sustancias alimenticias $(\mathrm{Zu}-$ nino 2000). Habiendo ejecutado la inversión requerida para el procesamiento de un aproximado del $30 \%$ de la producción lechera de la empresa caprina se obtuvo un monto de US\$ 1356 ( $\$ 685000$ ) (Cuadro 2). Este dato resulta mayor al compararse con INTI (2008) donde para los mismos bienes se estima una inversión de 260 mil colones (equivalencia a pesos argentinos: 1 US dólar / 0,22 pesos argentinos)

Dentro de la estructura de costos se detalla la adición de valor (Cuadro 3) sobre cada unidad producida y con el tamaño de lote especificado, permitiendo fijar precios convenientes que logren satisfacer la cadena
Cuadro 2. Costos de inversión efectuados para el procesamiento del dulce de leche de cabra. San José, Costa Rica. 2010.

\begin{tabular}{|c|c|}
\hline Equipo para procesamiento & Costo \\
\hline Recipiente 60 litros para cocción & $\$ 356,4(ф 180000,00)$ \\
\hline Refractómetro manual & $\$ 178,2(ф 90000,00)$ \\
\hline Utensilios varios & $\$ 128,7(\phi 65000,00)$ \\
\hline Acondicionamiento de cuarto & $\$ 297(\not 150000,00)$ \\
\hline Quemador de gas & $\$ 396(ф 200000,00)$ \\
\hline Total & $\$ 1356(\not 685000,00)$ \\
\hline
\end{tabular}

ya de por sí encarecida de este tipo de productos como lo es por ejemplo el rebajo en gastos por comisión de distribución en ventas estimados en un $10 \%$, principalmente por el hecho de representar nichos de mercado de mayor poder adquisitivo donde la decisión de consumo representa más la respuesta a un lujo alimentario que a una necesidad, con lo que, a nivel técnico se calificaría este producto como de carácter gourmet, del cual su mercado es heterogéneo y complejo, con escasos datos publicados (González 2009). Al no estar clasificados los productos gourmet de forma arancelaria, no existen estadísticas específicas de comercio para los productos englobados en este grupo de difícil delimitación.

Cuadro 3. Efecto de la adición de valor en el costo de cada unidad producida de dulce de leche de cabra. San José, Costa Rica. 2010. Nota: El precio de referencia para el flujo de caja utilizó el dato de $\notin 1515$ colones.

\begin{tabular}{lc}
\hline Presentación 275 ml & Valor \\
\hline Costo de ingredientes por unidad & $\$ 1,02(\phi 514,5)$ \\
Ingredientes + empaque & $\$ 1,81(\phi 914,5)$ \\
Ingredientes + empaque +energía & $\$ 2,17(\phi 1099,3)$ \\
Ingredientes + empaque +energía + mano & \\
de obra & $\$ 2,54(\phi 1284,1)$ \\
Costo más utilidad estimada (18\%) & $\$ 3,0(\varnothing 1515,3)$ \\
Precio a distribuidor (más un 8\%) & $\$ 3,3(\varnothing 1666,8)$ \\
Precio más el pago de impuesto & $\$ 3,72(\phi 1883,5)$ \\
Precio sug. expendedor (utilidad del 20\%) & $\$ 4,47(\phi 2260,0)$ \\
\hline
\end{tabular}


Debido a esta ambigüedad y falta de homogeneidad, se hace necesario enumerar como punto de partida las características que distinguen a un producto gourmet: 1. Calidad superior a sus homólogos en el mercado de masa, 2. distribución limitada a través de tiendas especializadas. Incluso si su distribución se realiza en supermercados, los productos gourmet son expuestos en forma separada, 3. producción limitada 4. Originalidad y unicidad, 5. Carácter regional o étnico, 6. Envasado único, distintivo, elaborado y de alta calidad, 7. Producto novedoso e impactante, 8. Precio superior y 9. Suelen poseer alguna certificación de denominación de origen o similar. (SCE 2009, González 2009). Lo anterior si bien representan inversiones agregadas y mayores costos productivos, se reflejan en el nivel de aceptación que podría ganar el dulce de leche (Encalada et al.2000). En el caso de nuestro país el agregarle valor podría mejorar la aceptación por productos caprinos que actualmente son concebidos más como "accesorios alimentarios" que como parte de la dieta del costarricense donde es necesario una mayor producción y mercadeo del producto; no solo para modificar la percepción sensorial que de la misma se tiene, sino también para incrementar su consumo. (Chacón et al. 2008).

Diseño de producto. El objetivo de la presentación y empaque del dulce de leche elaborado (Figura 1) fue transmitir puntualmente los conceptos mencionados en la metodología a un segmento del mercado turístico y/o especializado regional. Esta premisa se impone, inclusive, sobre el atributo de souvenir que caracteriza a los productos para turismo (Barbosa 2007).
El flujo de caja anual (Cuadro 4) desarrollado debió tomar en cuenta todos los relevantes para hacer éste lo más cercano a la realidad práctica proyectada. Lo anterior definió la inversión requerida, la estimación de producción mensual de dulce según el volumen definido, los requerimientos de mano de obra, calidad, así como todas las consideraciones respecto al manejo tecnológico para la conservación de la leche. El mismo sirvió como apoyo para realizar un análisis de sensibilidad en el cual se pudo valorar diferentes escenarios (cambios en: precios de venta unitarios (colones/litro), volúmenes de producción y cuotas del financiamiento) que a su vez modificaron los principales indicadores del proyecto (utilidad neta, tasa interna de retorno, valor presente neto).

La confección del proyecto técnico, la construcción y el montaje, contando con el capital de inversión vía crediticia, implicó un costo inicial por periodo de inmovilización de recursos (Castro 2001) considerablemente bajo, de tres meses, lo cual permitiría madurar económicamente el proceso y permitir una corriente financiera de liquidez en menor tiempo.

Los cálculos de proyección financiera, para su estimación incluyeron un crecimiento nulo anual de la producción, manteniéndose constante en 400 litros de leche procesada por mes (un $30 \%$ de la producción de leche del caprinocultor en el presente estudio). El monto de inversión planificado fue cubierto por un crédito de US\$ 1390 ( $\$ 700$ 000) al 12\% de interés anual durante un lapso definido de cinco años. Tomando en cuenta este valor, se obtuvo un dato actual neto (VAN) de US\$24 560 ( $₫ 12388000, ф 209$ por unidad),

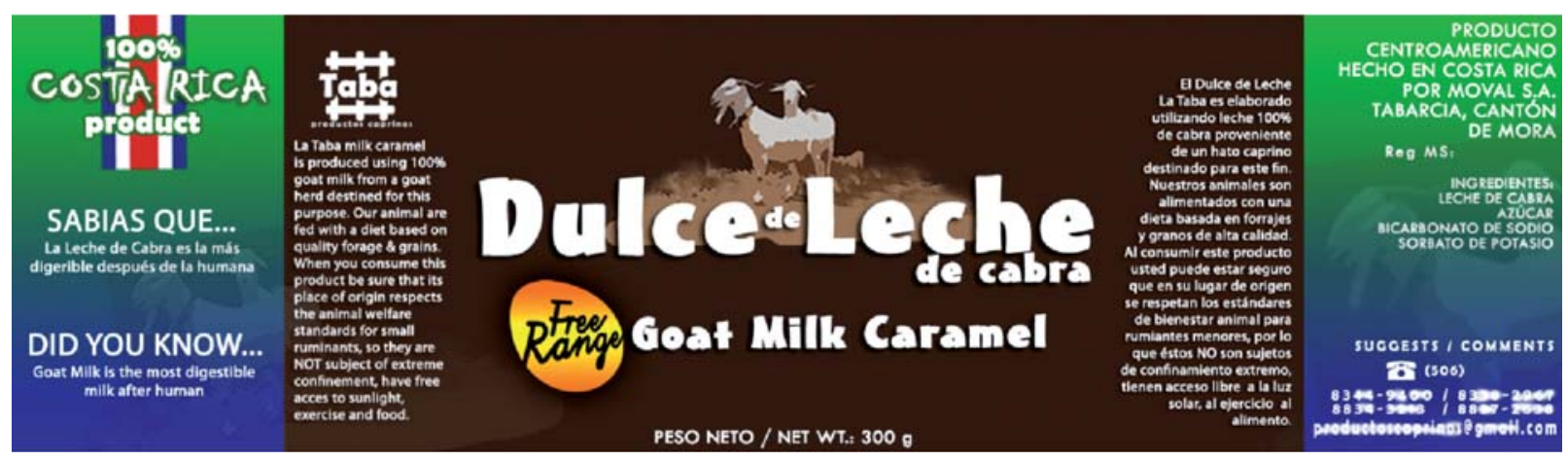

Figura 1. Ejemplo de etiqueta del dulce de leche de cabra (presentación peso neto: $275 \mathrm{ml}$ ) desarrollado como parte del proyecto. Diseño Gráfico: David Mora Valverde. San José, Costa Rica. 2010. 
Cuadro 4. Detalle de flujos de capital anual específicos para la producción de dulce de leche de cabra a partir de la compra de la materia prima directamente al productor. San José, Costa Rica. 2010.

\begin{tabular}{|c|c|c|c|c|c|c|}
\hline & Inversión(\$) & Año 1 & Año 2 & Año 3 & Año 4 & Año 5 \\
\hline Costo financiero & $\begin{array}{r}\$ 1356,00 \\
(\notin 685000)\end{array}$ & $\begin{array}{r}\$ 332,6 \\
(\notin 168000,0)\end{array}$ & $\begin{array}{r}\$ 332,6 \\
(\varnothing 168000,0)\end{array}$ & $\begin{array}{r}\$ 332,6 \\
(\notin 168000,0)\end{array}$ & $\begin{array}{r}\$ 332,6 \\
(\not 168000,0)\end{array}$ & $\begin{array}{r}\$ 332,6 \\
(\notin 168000,0)\end{array}$ \\
\hline Costo leche & & $\begin{array}{r}\$ 5227,7 \\
(\notin 2640000,0)\end{array}$ & $\begin{array}{r}\$ 5750,4 \\
(ф 2904000,0)\end{array}$ & $\begin{array}{r}\$ 6325,5 \\
(\notin 3194400,0)\end{array}$ & $\begin{array}{r}\$ 6958,0 \\
(\notin 3513840,0)\end{array}$ & $\begin{array}{r}\$ 7653,9 \\
(\notin 3865224,0)\end{array}$ \\
\hline Azúcar & & $\begin{array}{r}\$ 991 \\
(ф 500.487,80)\end{array}$ & $\begin{array}{r}\$ 1090,17(\phi 550 \\
536,59)\end{array}$ & $\begin{array}{r}\$ 1199,2(\phi 605 \\
590,24)\end{array}$ & $\begin{array}{r}\$ 1319,1 \\
(ф 666149,27)\end{array}$ & $\begin{array}{r}\$ 1451,0 \\
(\notin 732764,20)\end{array}$ \\
\hline Bicarbonato & & $\begin{array}{r}\$ 132,5 \\
(\not 66898,95)\end{array}$ & $\begin{array}{r}\$ 145,7 \\
(ф 73588,85)\end{array}$ & $\begin{array}{r}\$ 160,3 \\
(\notin 80947,74)\end{array}$ & $\begin{array}{r}\$ 176,3 \\
(\not 89042,51)\end{array}$ & $\begin{array}{r}\$ 193,9 \\
(\notin 97946,76)\end{array}$ \\
\hline Mano de obra & & $\begin{array}{r}\$ 2279,2 \\
(\not 1 \quad 152000,0)\end{array}$ & $\begin{array}{r}\$ 2463,6 \\
(\not 1244160,0)\end{array}$ & $\begin{array}{r}\$ 2690,2 \\
(\notin 1358576,00)\end{array}$ & $\begin{array}{r}\$ 2981,0 \\
(\phi 1505433,60)\end{array}$ & $\begin{array}{r}\$ 3279,1 \\
(\not 1655976,96)\end{array}$ \\
\hline Envases $275 \mathrm{ml}$ & & $\begin{array}{r}\$ 2592,2 \\
(\notin 1309090,91)\end{array}$ & $\begin{array}{r}\$ 2851,4 \\
(\not 1440000,0)\end{array}$ & $\begin{array}{r}\$ 3136,6 \\
(\not 1584000,0)\end{array}$ & $\begin{array}{r}\$ 3450,3 \\
(\notin 1742400,0)\end{array}$ & $\begin{array}{r}\$ 3795,3 \\
(\phi 1916640,0)\end{array}$ \\
\hline Etiquetado & & $\begin{array}{r}\$ 2345,3 \\
(\notin 1184415,58)\end{array}$ & $\begin{array}{r}\$ 2579,9 \\
(\notin 1302857,0)\end{array}$ & 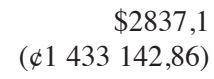 & $\begin{array}{r}\$ 3121,6 \\
(\notin 1576457,14)\end{array}$ & $\begin{array}{r}\$ 3433,8 \\
(\not 1734102,86)\end{array}$ \\
\hline Energía & & $\begin{array}{r}\$ 950,5 \\
(\not 480000,0)\end{array}$ & $\begin{array}{r}\$ 1045,5 \\
(ф 528000,0)\end{array}$ & $\begin{array}{r}\$ 1150,0 \\
(\$ 580800,00)\end{array}$ & $\begin{array}{r}\$ 1265,1 \\
(\not 638880,0)\end{array}$ & $\begin{array}{r}\$ 1391,6 \\
(\notin 702768,0)\end{array}$ \\
\hline Gastos anuales & & $\begin{array}{r}\$ 14853,2 \\
(\not 7500893,25)\end{array}$ & $\begin{array}{r}\$ 16259,6 \\
(\not 8211142,58)\end{array}$ & $\begin{array}{r}\$ 17852,3 \\
(\phi 9015456,84)\end{array}$ & $\begin{array}{r}\$ 19604,3 \\
(\not 9900202,52)\end{array}$ & $\begin{array}{r}\$ 21541,4 \\
(\notin 10878422,77)\end{array}$ \\
\hline $\begin{array}{l}\text { \# Unidades anuales - } \\
5 \% \text { pérdidas }\end{array}$ & & 5922 & 5922 & 5922 & 5922 & 5922 \\
\hline $\begin{array}{l}\text { Precio por unidad } \\
\text { vendida }\end{array}$ & & $\begin{array}{r}\$ 3,0 \\
\phi 1515,26)\end{array}$ & $\begin{array}{r}\$ 3,3 \\
(\notin 1666,79)\end{array}$ & $\begin{array}{r}\$ 3,63 \\
(\not 1833,46)\end{array}$ & $\begin{array}{r}\$ 3,99 \\
(ф 2016,81)\end{array}$ & $\begin{array}{r}\$ 4,39 \\
(\notin 2218,49)\end{array}$ \\
\hline Ingresos por venta & & $\begin{array}{r}\$ 17769,2 \\
(\notin 8973485,34)\end{array}$ & $\begin{array}{r}\$ 19546,2 \\
(\notin 9870833,87)\end{array}$ & $\begin{array}{r}\$ 21500,8 \\
(\notin 10 \quad 857917,26)\end{array}$ & $\begin{array}{r}\$ 23650,9 \\
(\notin 11943708,98)\end{array}$ & $\begin{array}{r}\$ 26015,9 \\
(\notin 13138079,88) \\
\end{array}$ \\
\hline
\end{tabular}

con un periodo de vida útil del mismo estimado en diez años, que resulta mayor comparado con Encalada et al. (2000) donde se estima un valor por unidad de US\$ 0,2 ( $\varnothing 101)$ para dulce de leche de vaca industrial de carácter comercial masificado, una presentación de menor peso y un TIR de 60,79\%. En comparación al valor obtenido en la presente investigación (226\%), el flujo por la venta del dulce de leche en presentación de $275 \mathrm{ml}$ es el responsable único para este ejercicio. Para llegar a los datos obtenidos de VAN y TIR se sugirió un margen mínimo de $18 \%$ por utilidad de venta del producto, lo cual adicionaría indirectamente un 39,5\% al margen de rentabilidad del productor por $\mathrm{kg}$ de leche transformado a dulce si se utiliza como base un costo de producción por $\mathrm{kg}$ de leche en Costa Rica de US\$ 0,9 ( $\$ 459)$ (Mora 2010).

Para el presente caso, dentro de la estructura de costos, se previó la disminución del porcentaje de utilidad hasta el $18 \%$ sugerido de tal manera que esto permitiese suficiente libertad a posteriores incrementos en los gastos de venta atribuibles al transporte y/o distribución (entre 5 y $8 \%$ encontrado para distribuidores de la zona) y por comisión de venta al detallista.

Del costo de producción de dulce leche obtenido (para lotes de 501 de leche), el rubro de mayor importancia en éste resultó la compra de la leche (Figura 2 ), con un $35 \%$ del costo total de producción, seguido por el envasado (18\%) y el etiquetado (16\%). El valor mínimo para producir un litro de dulce de leche (a "granel": sin etiqueta ni envase de vidrio) con las condiciones operativas establecidas fue de US\$ 6,46 (\$3265). El valor mínimo para producir cada unidad de presentación de $275 \mathrm{ml}$ (empacado) con las condiciones establecidas fue de US\$2,54 ( $₫ 1284)$, valor en el cual, si este fuese el precio de venta, la tasa interna de retorno se acercaría más a cero. Este dato resulta similar al reportado por Mendez (2011) donde estima un costo de US\$2,28 ( $\phi 1154)$ para una presentación 


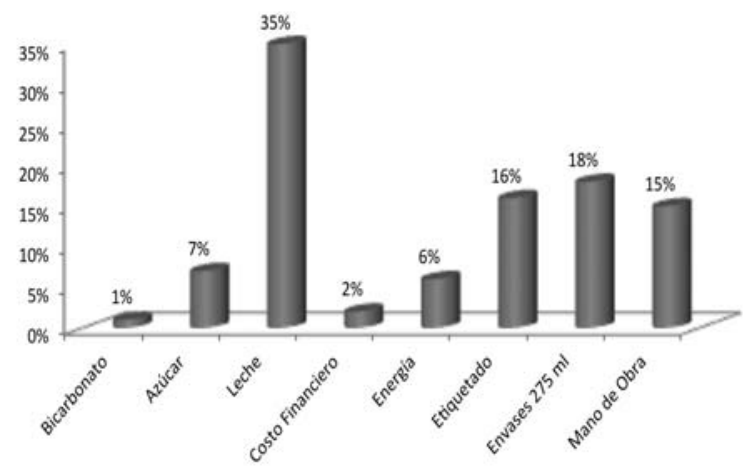

Figura 2. Distribución porcentual de los costos de producción por cada unidad de dulce de leche de cabra de 275 ml. San José, Costa Rica. 2010.

del mismo volumen $(275 \mathrm{ml})$ pero con empaque y presentación popular y cabe recalcar que no se toma en cuenta ningún costo financiero al precio del producto.

Respecto al análisis de rentabilidad, éste se convierte en un indicador fundamental para evaluar la eficiencia y eficacia económica de la unidad productiva y posteriormente para la implementación de estrategias que contribuyan a mejorar dicho sistema (Obando 2005). En este caso se analizó la rentabilidad financiera (ROE) la cual divide el beneficio neto entre el patrimonio neto y en términos generales relaciona el beneficio económico del productor con los recursos necesarios para obtener ese lucro, generando un dato de $17,9 \%$. Cabe tomar en cuenta que la mano de obra se estableció como contratación externa al sistema, por lo que si el mismo productor o el núcleo familiar se hiciese cargo del procesamiento, permitiría mejorar los beneficios de la actividad propuesta.

Dentro de los principales retos en Costa Rica para diversificar los productos caprinos, en este caso con el dulce de leche es definitivamente el esfuerzo en la comercialización, aspecto que dificulta la mejora productiva en tiempos más cortos. Es por ello que el caprinocultor debe ir más allá de la producción primaria y enfocarse en la búsqueda de mercados de mayor poder adquisitivo que permitan colocar al menos parte de su producción. Si es el caso de que algún productor tiene poco o nulo acceso a estos mecanismos articulados de comercialización, se recomienda buscar la orientación y apoyo en instituciones estatales dedicadas a este servicio.
No se debe confundir el indicador positivo de tasa interna de retorno obtenida como excesivamente promisoria ya que como indicador teórico que es, no refleja las posibilidades de aceptación de grandes volúmenes del producto. Por el contrario, permite retribuciones generosas pero equivalentes a una pequeña escala fijada en este proyecto. Igualmente cabe tomar en cuenta que bajo la estructura artesanal detallada, la inversión inicial resulta ser baja, aspecto que mejora el indicador mencionado.

Bajo las condiciones expuestas, la producción de dulce de leche de cabra hacia mercados selectivos en Costa Rica representa una oportunidad para mejorar la independencia empresarial del caprinocultor, esto debido a que la adición de valor a pequeños volúmenes de leche puede mejorar considerablemente el ingreso por unidad de leche producida.

\section{LITERATURA CITADA}

Benavides, H. 2007. Aplicación de métodos numéricos en el análisis financiero, Determinación de la TIR por el método de Newton Raphson. Universidad Técnica Particular de Loja, Ecuador. Consultado febrero 2009. Disponible en http://www.utpl.edu.ec/ucg/images/stories/papers/numericos\%20tir.pdf

Barbosa, M. 2007. La política del turismo cultura y el diseño de producto turístico para el turismo cultural. Caso: Corredor turístico Bogotá-Boyacá-Santander. Revista Escuela de Administración de Negocios. Número 060. Escuela de Administración de Negocios. Institución Universitaria. Bogotá, Colombia. p. 105-122.

Castillo, A; Taverna, M. 2000. Productos lácteos. Estrategias nutricionales para potenciar sus notables propiedades. Estación Experimental INTA Rafaela. Revista Chacra No837. p. 105.

Castro, M. 2001. El valor actual neto (VAN) como criterio fundamental de evaluación de negocios. Revista Economía y Desarrollo 128(1).

Chacón; A. 2004. Acidez y peso específico de la leche de cabra de un grupo de capricultores de la meseta central costarricense. Agronomía mesoamericana 15(2):179-183.

Chacón, A; Araya, Y; Gamboa, M; 2008. Percepciones y hábitos de consumo de la leche de cabra y sus derivados en los costarricenses. Agronomía mesoamericana 19(2):241-250. 
Corrales, J; Chacón, A. 2005. Estudio de opinión de consumidores sobre el queso fresco de cabra (Capra hircus) en Costa Rica. Rev. Agr. Trop. 35:39-49.

Encalada, D; Vélez, C; Mejía, M. 2009. Determinación de Costos de calidad del manjar de leche de la empresa D \& C. Tesis Escuela Superior Politécnica del Litoral. Ecuador (en línea). Consultado set. 2010. Disponible en http://www.dspace.espol.edu.ec/handle/123456789/1289

HFAC (Humane Farm Animal Care). 2005. Procedimientos estándares para el cuidado de los animales de Granja. Estándar de cuidado de Ganado Ovino. Hendon, VA. U.S.A. p. 6-10

Instituto Nacional de Tecnología Industrial (INTI). 2008. Elaboración de dulce de leche, instructivo para unidades de producción. Argentina. (en línea). Consultado abril 2010. Disponible en http://www.inti.gob.ar/ extension/cuadernillos/pdf/dulcedeleche.pdf

Mora, D. 2010. Estudio bioeconómico para el establecimiento de una explotación caprina en Costa Rica. Universidad de Costa Rica, Costa Rica. Agronomía Mesoamericana 21(1):113-120.

MEIC (Ministerio de Economía, Industria y Comercio). 2002. Reglamento RTC 100 Etiquetado de alimentos preenvasados. Costa Rica (en línea). Consultado abril 2011. Disponible en http://www.reglatec.go.cr/decretos/26012.pdf.

MEIC (Ministerio de Economía, Industria y Comercio). 2002. Reglamentación técnica costarricense. Centro de Información de Obstáculos Técnicos del comercio. Costa Rica (en línea). Consultado abril 2011. Disponible en http://www.reglatec.go.cr/

Mendez, S. 2011. Efecto de distintas proporciones de leche de cabra y leche de vaca sobre las características físicas y sensoriales de un dulce de leche. Proyecto de graduación escuela de Tecnología de Alimentos. Universidad de Costa Rica. San José, Costa Rica. p. 4.

Nastasi, V. 2007. Gestión estratégica de los costos agropecuarios. Universidad de Buenos Aires, Argentina.
Consultado 5 mayo 2009. Disponible en http://www. intercostos.org/documentos/Nastasi.pdf

Obando, G. 2005. Análisis de rentabilidad en sistemas de producción lechera en ganadería de altura: Caso Finca Las Josefinas. IX Seminario de Pastos y Forrajes 2005. Consultado 11 setiembre 2009. Disponible en http:// avpa.ula.ve/eventos/ix_seminario_pastosyforraje/ Talleres/T4-GuidoObando.pdf

Sabadzija, GN; González, MF. 2007. Análisis económico de un modelo de producción de leche caprina en sistema intensivo bajo riego, en Catamarca, Argentina (en línea). Catamarca, Argentina. Consultado 2 junio 2009. Disponible en http://www.produccionbovina. com/produccion_caprina/produccion_caprina/87sabadzija.htm

SCE (Secretaría de Comercio Exterior). 2009. Informe del Sector Gourmet. Chubut, Patagonia. Argentina. Consultado 2 junio 2011. Disponible en http:// www.chubutalmundo.gov.ar/comercio/INFORME_ SECTOR_GOURMET.pdf

SE (Secretaría de Economía Mexicana). 2005. Proyecto de Norma Oficial Mexicana: Cajeta, dulce de cajeta, denominación, especificaciones fisicoquímicas, información comercial y métodos de prueba. México. Consultado febrero 2011. Disponible en http://www.sagarpa.gob.mx/ganaderia/Publicaciones/ Lists/Sistemas \% 20 Productos\%20 Pecuarios/ Attachments/91/PROYNOCAJETA2005.pdf

Vilaboa, Julio, Diaz, P; Platas, D. 2006. Productividad y autonomía en sistemas de producción ovina: Dos propiedades emergentes de los agroecosistemas. INCI 31(1):37-44.

Velasco, R; Gonzales, J; Jahn, E. sf. Producción de cabras lecheras. Capítulo 11.p. 172

Zunnino, A. 2000. Dulce de leche, aspectos básicos para su adecuada elaboración. Departamento de Fiscalización de Industrial Lácteas. Ministerio de Agricultura y Ganadería. Argentina. p. 5. 